\title{
Compressed collagen constructs with optimized mechanical properties and cell interactions for tissue engineering applications
}

Ajalloueian, Fatemeh; Nikogeorgos, Nikolaos; Ajalloueian, Ali; Fossum, Magdalena; Lee, Seunghwan; Chronakis, loannis S.

Published in:

International Journal of Biological Macromolecules

Link to article, DOI:

10.1016/j.ijbiomac.2017.11.117

Publication date:

2018

Document Version

Peer reviewed version

Link back to DTU Orbit

Citation (APA):

Ajalloueian, F., Nikogeorgos, N., Ajalloueian, A., Fossum, M., Lee, S., \& Chronakis, I. S. (2018). Compressed collagen constructs with optimized mechanical properties and cell interactions for tissue engineering applications. International Journal of Biological Macromolecules, 108, 158-166.

https://doi.org/10.1016/j.ijbiomac.2017.11.117

\section{General rights}

Copyright and moral rights for the publications made accessible in the public portal are retained by the authors and/or other copyright owners and it is a condition of accessing publications that users recognise and abide by the legal requirements associated with these rights.

- Users may download and print one copy of any publication from the public portal for the purpose of private study or research.

- You may not further distribute the material or use it for any profit-making activity or commercial gain

- You may freely distribute the URL identifying the publication in the public portal 


\section{Accepted Manuscript}

Title: Compressed collagen constructs with optimized mechanical properties and cell interactions for tissue engineering applications

Authors: Fatemeh Ajalloueian, Nikolaos Nikogeorgos, Ali Ajalloueian, Magdalena Fossum, Seunghwan Lee, Ioannis S.

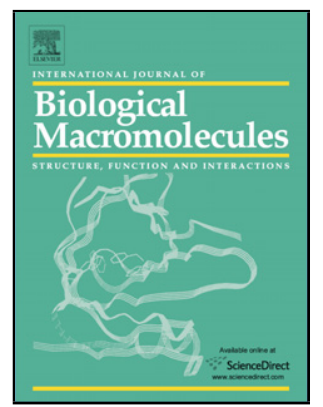

Chronakis

PII:

DOI:

Reference:

S0141-8130(17)30946-7

https://doi.org/10.1016/j.ijbiomac.2017.11.117

BIOMAC 8597

To appear in: $\quad$ International Journal of Biological Macromolecules

Received date: $\quad 14-3-2017$

Revised date: $\quad 16-11-2017$

Accepted date: $\quad$ 17-11-2017

Please cite this article as: Fatemeh Ajalloueian, Nikolaos Nikogeorgos, Ali Ajalloueian, Magdalena Fossum, Seunghwan Lee, Ioannis S.Chronakis, Compressed collagen constructs with optimized mechanical properties and cell interactions for tissue engineering applications, International Journal of Biological Macromolecules https://doi.org/10.1016/j.ijbiomac.2017.11.117

This is a PDF file of an unedited manuscript that has been accepted for publication. As a service to our customers we are providing this early version of the manuscript. The manuscript will undergo copyediting, typesetting, and review of the resulting proof before it is published in its final form. Please note that during the production process errors may be discovered which could affect the content, and all legal disclaimers that apply to the journal pertain. 


\title{
Compressed collagen constructs with optimized mechanical properties and cell interactions for tissue engineering applications
}

Fatemeh Ajalloueian $^{1 *}$, Nikolaos Nikogeorgos², Ali Ajalloueian ${ }^{3}$, Magdalena Fossum ${ }^{4,5}$, Seunghwan Lee $^{2}$, Ioannis S Chronakis ${ }^{1}$

1. Nano-BioScience Research Group, DTU-Food, Technical University of Denmark, DK-2800 Kgs. Lyngby, Denmark

2. Department of Mechanical Engineering, Technical University of Denmark, DK-2800 Kgs. Lyngby, Denmark

3. Department of Materials Engineering, Isfahan University of Technology, Isfahan, 8415683111, Iran

4. Department of Women's and Children's Health, Karolinska Institutet, Stockholm, Sweden.

5. Department of Paediatric Surgery, Astrid Lindgren Children's Hospital, Karolinska University Hospital, Stockholm, Sweden

Corresponding author: Fatemeh Ajalloueian (faaj@food.dtu.dk)

\section{Highlights}

- We are introducing a simple method to obtain compressed collagen sheets with improved fibrillar density

- Decreasing the collagen concentration in initial hydrogel formula, results in collagen sheets with lower collagen densities compared to high collagen density in conventional compressed sheets.

- The lower collagen density in compressed collagen sheets, the higher proliferation and metabolic activity of fibroblasts, as well as increased elasticity.

\begin{abstract}
In this study, we are introducing a simple, fast and reliable add-in to the technique of plastic compression (PC) to obtain collagen sheets with decreased fibrillar densities, representing improved cell-interactions and mechanical properties. Collagen hydrogels with different initial concentrations (1.64 $\mathrm{mg} / \mathrm{mL}$ to $0.41 \mathrm{mg} / \mathrm{mL}$ ) were compressed around an electrospun sheet of PLGA. The scaffolds were then studied as non-seeded, or seeded with 3T3 fibroblast cells and cultured for 7 days. Confocal microscopy and TEM imaging of non-seeded scaffolds showed that by decreasing the share of collagen in the hydrogel formula, collagen sheets with similar thickness but lower
\end{abstract}


fibrous densities were achieved. Nanomechanical characterization of compressed collagen sheets by AFM showed that Young's modulus was inversely proportional to the final concentration of collagen. Similarly, according to SEM, MTS, and cell nuclei counting, all the scaffolds supported cell adhesion and proliferation, whilst the highest metabolic activities and proliferation were seen in the scaffolds with lowest collagen content in hydrogel formula. We conclude that by decreasing the collagen content in the formula of collagen hydrogel for plastic compression, not only a better cell environment and optimum mechanical properties are achieved, but also the application costs of this biopolymer is reduced.

\section{Key words}

Collagen hydrogel, Plastic compression, Fibrillar density, Fibroblast, Proliferation, Infiltration, Nanomechanical characteristics

\section{Introduction}

Biocompatible scaffold systems as cell delivery vehicles play an important role in tissue repair and regeneration. Among various forms of scaffold materials, naturally derived polymers possess outstanding characteristics (i.e. biocompatibility, low immunogenicity, capability of being naturally remodeled by cells) for tissue regeneration applications [1]. Collagen is a natural polymer with extensive utilization in regenerative medicine. Collagen type I is the most commonly used collagen due to its abundance, ubiquity, and biocompatibility [2,3]. Hydrogel scaffolds of collagen, although very conventional and capable of rapid introduction of cells, suffer from low mechanical strength. It (the low mechanical strength) originates from low protein to water ratio [4], leading to smaller collagen fibril density in collagen hydrogels compared to their natural equivalent [5]. Moreover, collagen hydrogels (or hyper hydrated gels) are unstable materials, as they undergo selfcompression under their own weight as soon as transferring a freshly cast hydrogel to another surface (glass surface or a blotting set) causing the liquid to expel out of the hydrogel [6]. To increase fibrillar density of collagen-based scaffolds and/or improve their mechanical properties, researchers have performed different studies including 3-D microfabrication methods in collagen matrices [7,8], crosslinking [9], or making high concentration collagen scaffolds of around 10-45 $\mathrm{mg} / \mathrm{ml}$ via ultracentrifugation [10] or evaporation methods [11]. While these methods are either complicated (3-D microfabrication methods) or can be toxic to cells (crosslinking procedures), the technique of plastic compression (PC) of collagen hydrogels [6] has attracted a great deal of attention for tissue engineering studies $[4,5,12-18]$. In this procedure, which was introduced by 
Brown and colleagues in 2005 [6], excess water of the collagen hydrogel is removed via mechanical compression, leading to a denser and stronger construct compared to conventional gels. This technique has been applied in different fields of tissue engineering and regenerative medicine such as in the cornea [12,14], tendon [19], bone [4], and urinary bladder [16,20] regeneration. The collagen sheet obtained from PC method, although stronger than conventional collagen hydrogels, still suffers from handling difficulties [17] and is not strong enough for some specific tissue engineering applications in need of high mechanical strength and/or if exposed to frequent cycles of loading and unloading [16,17]. To improve the mechanical properties of PC sheets, multilayer fabrication through stacking, rolling, or serial compression of PC sheets have been offered by different research groups [3,4,6]. Another solution is to perform the plastic compression over a supporting substrate so that a hybrid construct including collagen can be achieved [16-18]. In this way, the supporting substrate (usually a synthetic polymeric sheet) is responsible for fulfilling the mechanical requirements and the PC collagen provides the cells with a fibrous protein construct similar to natural connective tissue. Such hybrid constructs have shown satisfactory outcomes from both cell behavior and mechanical aspects [16-18], and have the potential to be implemented for tissue engineering purposes with specific requirements of layered cell seeding and high mechanical strength.

While PC is considered as a technique leading to very dense fibrillar structures[3,4,21], we address a question in this study whether we could rather reduce the collagen fibrillar density in compressed sheets of collagen in the context of further optimizing the cellular interaction with the final scaffold. On the other hand, although there have been some studies so far about optimum collagen concentration in collagen hydrogels applied for tissue engineering [11,22,23], the effect of collagen concentration (lower concentrations compared to conventional collagen concentration applied for making PC sheets) on the overall mechanical and cell interaction behavior of compressed collagen sheets has yet to be defined. Hence, in this study we have offered some modifications to the conventional hydrogel formula applied for PC, aiming to achieve less dense but still strong collagen constructs. To this end, we have applied different formulae for collagen hydrogel preparation, compressed the gels onto supporting substrate (here PLGA electrospun sheet), and compared the final constructs from different aspects including: internal collagen micro-architecture, mechanical properties, and cell-scaffold behaviors including cell attachment, and proliferation. The reason we applied PLGA electrospun sheet as the supporting substrate was to enhance the mechanical properties of the whole scaffold, and to further develop the PC collagen-PLGA- PC collagen 
layered scaffolds applied in our previous studies[18]. Herein, we have focused on improving the functional properties of the collagen layer, and believe the modifications introduced in this study can be implemented in other hybrid scaffolds applying PC collagen.

\section{Materials and methods}

\subsection{Preparation of the scaffold constructs}

Four different collagen hydrogels were prepared according to the quantities mentioned in Table 1 and the protocols described earlier [6,17]. In brief, sterile rat-tail collagen type I solutions $(2.06$ $\mathrm{mg} / \mathrm{mL}$ protein in $0.6 \%$ acetic acid; First Link Ltd, UK) were mixed with $10 \%$ Eagle's minimum essential medium (MEM; Invitrogen, Denmark), and neutralized with 2.5 M NaOH. Finally, alphaMEM medium was added. Hydrogels were labeled as C80, C60, C40, and C20. For instance, C80 was the symbol for the conventional collagen hydrogel used for plastic compression in which $80 \%$ volumetric share of the hydrogel was made up of collagen with the original concentration of 2.06 $\mathrm{mg} / \mathrm{mL}$. In other words, C80 was equivalent to the hydrogel with collagen concentration of 1.64 $\mathrm{mg} / \mathrm{mL}$. It is worth noting that MEM $10 \mathrm{X}$ is the indicator for neutralization of collagen solution due to the change in color of phenol red dye in media. Therefore, the volumetric ratio of MEM 10X to collagen solution has been adjusted to be kept around $12.5 \%$ of collagen volume (similar to the ratio in conventional hydrogel formula).

Table 1: formulations to prepare different collagen hydrogels for plastic compression

\begin{tabular}{llll}
\hline \multirow{2}{*}{ Collagen hydrogel formula } & \multicolumn{3}{l}{ Volumetric percentage (v/v\%) } \\
\cline { 2 - 4 } & Collagen & MEM 10X & Alfa-MEM \\
\hline C80 $(1.64 \mathrm{mg} / \mathrm{mL}$ collagen in final hydrogel $)$ & 80 & 10 & 10 \\
C60 $(1.23 \mathrm{mg} / \mathrm{mL}$ collagen in final hydrogel $)$ & 60 & 7.5 & 32.5 \\
C40 $(0.82 \mathrm{mg} / \mathrm{mL}$ collagen in final hydrogel $)$ & 40 & 5 & 55 \\
C20 $(0.41 \mathrm{mg} / \mathrm{mL}$ collagen in final hydrogel $)$ & 20 & 2.5 & 77.5 \\
\hline
\end{tabular}

$1 \mathrm{~mL}$ of each of the prepared collagen hydrogels was cast into circular shaped molds (diameter of $34 \mathrm{~mm}$ ) and were incubated at $37^{\circ} \mathrm{C}$ for $20 \mathrm{~min}$ to undergo the gelation procedure. After that, PLGA electrospun sheet (with optimized structure as described earlier [18], average fiber diameter of $705 \mathrm{~nm}$, and average thickness of $80 \mu \mathrm{m}$ ) was added onto the collagen gel, and was covered with 
another $1 \mathrm{~mL}$ of the same type of collagen hydrogel. After 20 minutes, the hybrid construct was transferred onto blotting elements, consisting of a layer of sterile $110 \mathrm{~mm}$-thick nylon mesh ( 40mm mesh size) and a sterile 400-mm-thick stainless steel mesh (mesh size $\sim 200 \mathrm{~mm}$ ), which were placed on top of three sterile gauze pads (Fig. 1). The set gels were covered with a second nylon mesh and a loading plate (as a static weight) (120 g) for $5 \mathrm{~min}$ at room temperature, leading to the formation of hybrid constructs of PCX-PLGA-PCX; X was any of the values 80, 60, 40 or 20, representing the different collagen concentrations. PC80 (as an example) represents the C80 after experiencing the plastic compression procedure.

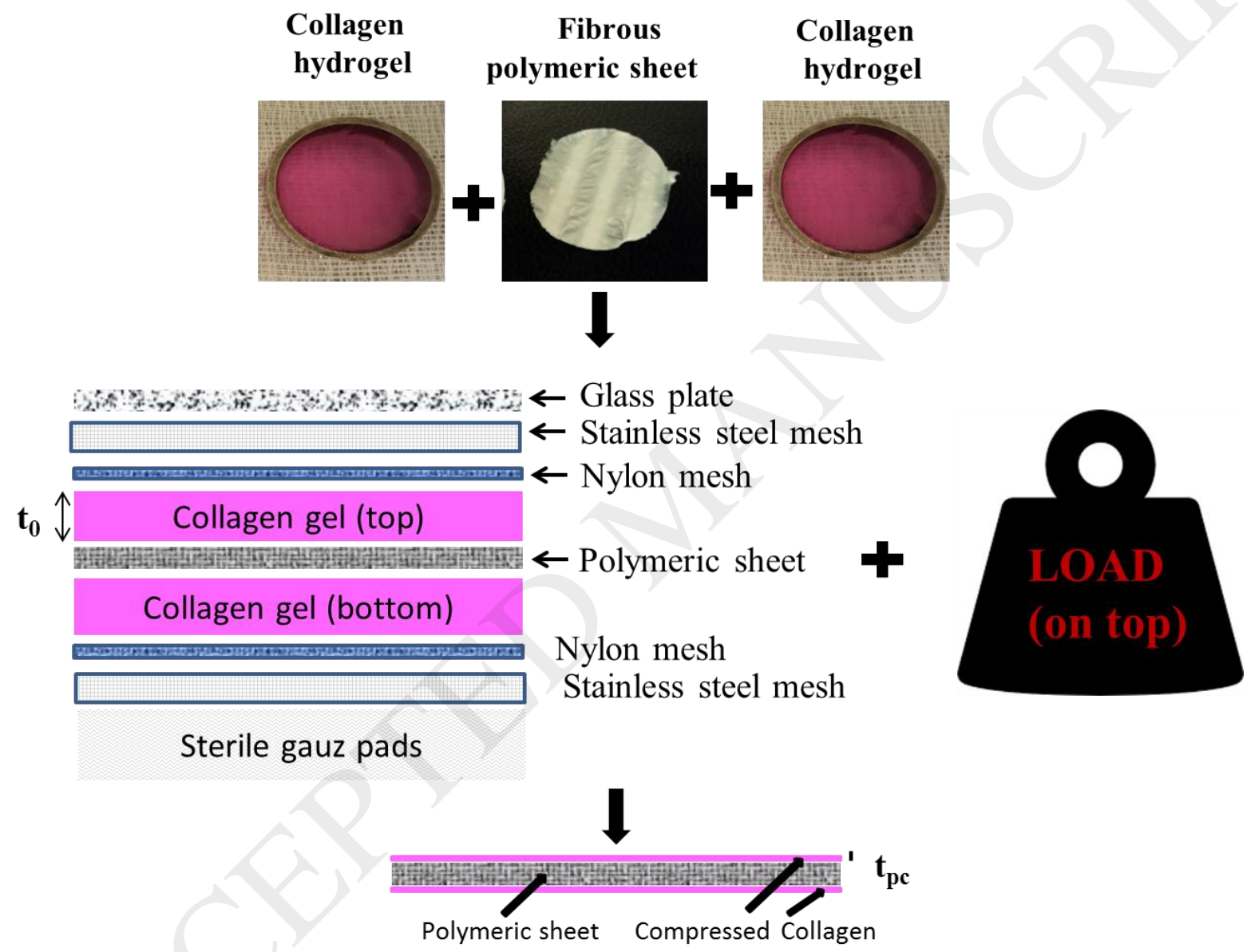

Fig. 1. A schematic diagram showing layered structure of polymeric sheet and collagen gels. The diagram shows the compression setup and different thickness of the collagen layers before $\left(\mathrm{t}_{0}\right)$ and after plastic compression $\left(t_{\mathrm{pc}}\right)$

\subsection{Scaffold characterization}

\subsubsection{Calculation of collagen concentration in PC sheets}


If we assume that only water is expelled during compression of collagen hydrogel, we can calculate the theoretical collagen concentration in different hydrogels undergoing compression by measuring the initial volume of hydrogel and the volume of the final sheet based on the rule of no change in collagen mass before or after compression.

$c_{p c}=\frac{c_{0} \times t_{0}}{t_{p c}} \quad$ Equation (1)

where $c_{0}$ represent the concentration of collagen in the initial hydrogel, $t_{0}$ the thickness of initial gel and $c_{p c}$ and $t_{p c}$ the concentration and thickness of the compressed sheets, respectively.

\subsubsection{Morphological characterization of hybrid constructs}

\section{Scanning Electron Microscopy}

The morphology of electrospun nanofibers, compressed collagen sheets, and hybrid constructs of PCX-PLGA-PCX was studied with scanning electron microscopy (SEM). Specimens were fixed in $2.5 \%$ glutaraldehyde in cacodylate buffer overnight and then washed with cacodylate buffer. Subsequently, samples were dehydrated with increasing concentrations of ethanol $(30 \%, 50 \%, 70 \%$, 90\%, and 100\%) for 10 min each. Finally, the constructs were treated with hexamethyldisilazane (HMDS) for further water extraction. The dehydrated constructs were maintained in desiccators equipped with vacuum for overnight air-drying. After sputter-coating with platinum, Quanta FEG 3D scanning electron microscope (SEM) was used to observe surface of scaffolds. The average fiber diameter of the scaffolds was measured by applying ImageJ 1.46R (NH, Maryland, USA) to the SEM micrographs.

\section{Laser scanning confocal microscopy}

To visualize the 3D architecture of the collagen-PLGA nano-micro network, PCX-PLGA-PCX constructs were assessed by LSM 700 confocal laser scanning microscope (Carl Zeiss, Germany) after staining PLGA with rhodamine B, and collagen with Alexa Fluor ${ }^{\circledR} 488$ reactive dye (Alexa Fluor® 488 Protein Labeling Kit). Fluorescent Rhodamine B was added to the PLGA solution (prepared for electrospinning) so that the rhodamine concentration in the PLGA solution was 50 $\mu \mathrm{g} / \mathrm{mL}$. The solution was stirred for extra 1 hour before starting electrospinning. For collagen staining, we used a vial of reactive dye (Alexa Fluor® 488 Protein Labeling Kit) for labeling each 8 
$\mathrm{mL}$ of collagen solution $(2.06 \mathrm{mg} / \mathrm{mL})$. Having mixed the collagen solution with reactive dye, the mixture was stirred for 10 minutes to dissolve the dye. For all different collagen concentrations, the hydrogel preparation procedure was performed using the stained collagen. The hybrid PC-PLGAPC collagen scaffolds were then observed by laser scanning confocal microscopy (LSM 700, Carl Zeiss, Germany). The excitation wavelengths for rhodamine B and the Alexa Flour 488 reactive dye were 559 and $519 \mathrm{~nm}$ respectively, and multi-track images were captured with a 63x/1.40NA objective.

\section{Tensile mechanical properties}

Tensile properties of the hybrid scaffolds (PCX-PLGA-PCX; Where X is 80, 60, 40 or 20) were determined using a Texture Analyzer (TA.XT plus; Stable micro systems Ltd, UK) fitted with a calibrated load cell of $5 \mathrm{~kg}$. Specimens were tested at a crosshead speed of $10 \mathrm{~mm} / \mathrm{min}$ and a gauge length of $40 \mathrm{~mm}$ under wet conditions at $25^{\circ} \mathrm{C}$. Samples were prepared by incubating the hybrid constructs in PBS for $24 \mathrm{~h}$ (after preparation) at $37^{\circ} \mathrm{C}$ and then cut in rectangular shape (10mm $\mathrm{x}$ $6 \mathrm{~mm}$ ). Dimensions were measured using calibrated Mitutoyo CS callipers and were entered to the instrument software. Force and displacement were recorded during testing. Stress/strain curves for each specimen were plotted and the tensile strength (fracture stress), maximum strain and elastic modulus were determined.

\subsubsection{Nanomechanical properties of compressed collagen layer: AFM analyses}

Mechanical properties of the scaffolds were further investigated at a microscale using atomic force microscopy (AFM). To this end, PCX-PLGA-PCX was prepared as mentioned above. Immediately after compression, the top compressed collagen layer was manually separated from the PLGA sheet and placed onto a silicon wafer. Samples were kept wet until the test was performed. Dimension Edge AFM (Bruker, Santa Barbara, CA) was employed and force-distance measurements were carried out in phosphate buffered saline (PBS). A rectangular-shaped silicon tip (model NP, Bruker) with normal spring constant of $2.8 \mathrm{~N} / \mathrm{m}$ was employed throughout all AFM experiments. A polyethylene microsphere (PE, Cosheric, CPMS-0.096, Santa Barbara, CA) with the diameter of 56 $\mu \mathrm{m}$ was attached using epoxy glue (DANA LIM, Epoxy Universal 335, Denmark) at the end of cantilever. The normal spring constant of the cantilevers was obtained with the generalized Sader method with a correction of the tip/sphere offset [24,25]. Nanomechanical analysis of the spherical indentation was carried out according to the JKR model of a sphere on a flat [26]: 
$\delta(\alpha)=\frac{\alpha^{2}}{R}-\left(\frac{2 \pi \gamma a}{E^{*}}\right)^{1 / 2}$

Equation (2)

$F(\alpha)=\frac{4 E^{*} \alpha^{3}}{3 R}-2\left(2 \pi E^{*} \gamma \alpha^{3}\right)^{1 / 2} \quad$ Equation (3)

where, $\alpha$ represents the contact radius of the materials and $R$ the radius of the indenter (PE). Solving the above system of equations ( 2 and 3 ) along the contact part of the force versus indentation curve provided the values of $\alpha$ and $E^{*}$. Since the Young moduli of PE were several orders of magnitude higher than that of the sample, the extracted $E$ from spherical indentation analysis could be reduced as: $E \approx E^{*} \cdot\left(1-v_{\mathrm{s}}^{2}\right)$, where $v_{\mathrm{s}}$ represents the Poisson ratio of the sample, and $E^{*}$ its Young modulus [27-29]. Conversion of force versus piezo displacement curves into force versus indentation/spheresample distance was achieved by subtracting the cantilever deflection from the piezo displacement. All data analysis was performed using homemade software written in Matlab (The Mathworks, Inc. Novi, MI). The data of insets in all figures were plotted in Origin (OriginLab, Northampton, MA).

\subsection{Cell studies}

\subsubsection{Cell Culture of $3 T 3$ Fibroblasts}

NIH 3T3 mouse fibroblasts (ATCC, U.K.) were cultured in DMEM (Dulbecco's ModifiedEagle Medium, Gibco, USA) containing 10\% fetal bovine serum (Invitrogen, Life Technologies, Paisley, UK), 50U/mL penicillin, and 50U/mL streptomycin (Sigma, Denmark). The medium was replaced every 3 days and cultures were maintained in a tissue culture incubator at $37{ }^{\circ} \mathrm{C}$ with $5 \%$ $\mathrm{CO}_{2}$. After reaching about $80 \%$ confluence, cells were detached by $0.05 \%$ trypsin/0.05\% EDTA. Cells were seeded onto the scaffolds at a density of 5,000 cells $/ \mathrm{cm}^{2}$, and were cultured in incubator at $37^{\circ} \mathrm{C}$ with $5 \% \mathrm{CO}_{2}$.

\subsubsection{Metabolic Activity and Proliferation of 3T3 Fibroblasts.}

Cell viability and metabolic activity in response to different substrates of PC80-PLGA-PC80, PC60-PLGA-PC60, PC40-PLGA-PC40 and PC20-PLGA-PC20 were measured using MTS cytotoxicity assay (CellTiter 96 AQueous Non-Radioactive Cell Proliferation Assay, Promega, Madison, WI, USA), according to the manufacturer's instructions. Cells were seeded at a density of 5,000 cells $/ \mathrm{cm}^{2}$, and their metabolic activity was evaluated during a 7-day period. On days 1, 4, and 7, the cell-seeded sheets were cut with a punch biopsy tool of $10 \mathrm{~mm}$ diameter (fitting the 24 well plates), and transferred to 24 well plates, after washing with PBS. Thereafter, $500 \mu \mathrm{L}$ of culture 
medium and $100 \mu \mathrm{L}$ of MTS solution were added to each well. Cells were maintained for an additional 4 hours in a humidified incubator at $37{ }^{\circ} \mathrm{C}$ and $5 \% \mathrm{CO}_{2}$. Then $100 \mu 1$ of the solution in each well was transferred to a 96 well plate, and the absorbance was read at $490 \mathrm{~nm}$ in a spectrophotometer Microplate Reader (wallac VICTOR3, 1420 multilable counter, PerkinElmer, MA). Non-seeded scaffolds were used as negative controls, and the measured absorbance was defined as cell metabolic activity on each substrate at different time points. This was done in triplicates followed by calculations of mean values and standard deviations.

Proliferation of cells was determined by counting the number of DAPI-stained cell nuclei on different scaffolds. Cell nuclei were counter-stained with 4,6-diamidino-2-phenylindole dihydrochloride (DAPI, Sigma-Aldrich; $1 \mu \mathrm{g} / \mathrm{mL}$ ) in different samples per time points of 1 , and 7 days. A minimum of 5 randomly selected visual fields (at $20 \times$ magnification in a confocal laser scanning microscope, Zeiss LSM 700) were studied.

\subsubsection{Cell Morphology}

Morphological studies of 3T3 fibroblasts grown on PCX-PLGA-PCX sheets were performed after 7 days of cell culture by SEM. Sample preparation was performed as stated earlier (2.2.1). SEM was used to observe cell attachment and cell morphology on the scaffolds.

\subsubsection{D Cell distribution}

3D architectural study of 3T3 fibroblasts grown on PC sheets was performed after 7 days of cell culture by laser scanning confocal microscopy. Cell-seeded constructs were washed in PBS and subsequently fixed in $4 \%$ formaldehyde (Sigma-Aldrich, Denmark) for $10 \mathrm{~min}$. They were washed and stained for 30 min with Phalloidin (Molecular Probes, Denmark) diluted in PBS/0.1\% Triton X100 (2 U/ ml) (Sigma-Aldrich, Denmark), followed by counterstaining with 40,60-diamidino-2phenylindole (DAPI, Sigma-Aldrich, Danmark). Stained cells were visualized by Zeiss LSM 700 confocal laser scanning microscope and ZEN software (Carl Zeiss, Germany). Multi-track images were captured with a 40x objective, and cell distributions were evaluated through an orthogonal observation.

\subsection{Statistical analysis}


Graphpad Prism 6 (Graphpad software, CA, USA) was used to do statistical analyses. Significance levels were shown as “*” for $p<0.05$, "**” for $p<0.01$, "***” for $p<0.001$ and "****” for $p<$ 0.0001. All data were compared by using an unpaired t-test.

\section{Results and discussion}

\subsection{Scaffold characterization}

\subsubsection{Collagen content and spatial density of fibers in compressed sheets}

In conventional PC procedures, typically a collagen hydrogel with starting concentration of around $2 \mathrm{mg} / \mathrm{mL}$ is prepared, solidified by the process of fibrillogenesis [30], and compressed to remove excess water. In this way, more than 90 percent of the volume of hydrogel (water) is expelled, and the concentration of collagen undergoes a huge increase to around $300 \mathrm{mg} / \mathrm{mL}$ in final compressed sheet. Such a high concentration not only limits the application of plastic compressed collagen sheets due to its cost, but also is prone to trap the cells (if seeded inside the hydrogel) or restrict their migration or infiltration into the scaffold (if seeded on top of the construct).

Thus, it is desirable if the formula of collagen hydrogel can be modulated in the preparation for plastic compression so that the compression procedure would lead to sheets with lower density of collagen than typical values mentioned above. In this study, we attempted to achieve this goal by preparing collagen hydrogels with initially lower concentrations as shown in Table 1. The conventional collagen hydrogel formula for plastic compression $(\mathrm{C} 80)$ contained $80 \%$ volumetric collagen with concentration of $2.06 \mathrm{mg} / \mathrm{mL}, 10 \%$ volumetric MEM 10X, and remaining volume as alpha-MEM (10\% v). The other solutions we prepared in this study included $60 \%, 40 \%$ and $20 \%$ volumetric collagen (Table1) in the primary hydrogel and are dented as C60, C40, and C20, respectively. MEM $10 \mathrm{X}$ was used as $12.5 \%$ of the collagen volume in each formula and the remaining volume was alpha-MEM. We prepared hybrid constructs of collagen-PLGA-collagen (Fig. 1), using C80 to $\mathrm{C} 20(1.64 \mathrm{mg} / \mathrm{mL}$ in $\mathrm{C} 80$ to $0.41 \mathrm{mg} / \mathrm{mL}$ in $\mathrm{C} 20)$, and performed compression on the whole constructs.

To study the effect of compression on different PCX-PLGA-PCX constructs, we analyzed the fiber density of collagen matrices using confocal microscopy. The confocal images in Fig. 2(a-d) showed the internal structure of top compressed collagen sheets (PC80, PC60, PC40, and PC20). Fig. 2a demonstrated that compressing C80 over PLGA led to a very dense intertwined mesh of collagen fibers (PC80). Figs. 2b-d also revealed that decreasing the initial collagen concentration (to C60, C40, and then C20) resulted in decreasing spatial density of fibers. Consequently, PC20 (Fig. 2d) 
represented a porous construct with the lowest density of fibrous network, a trend that was expected based on previous reports [31,32].
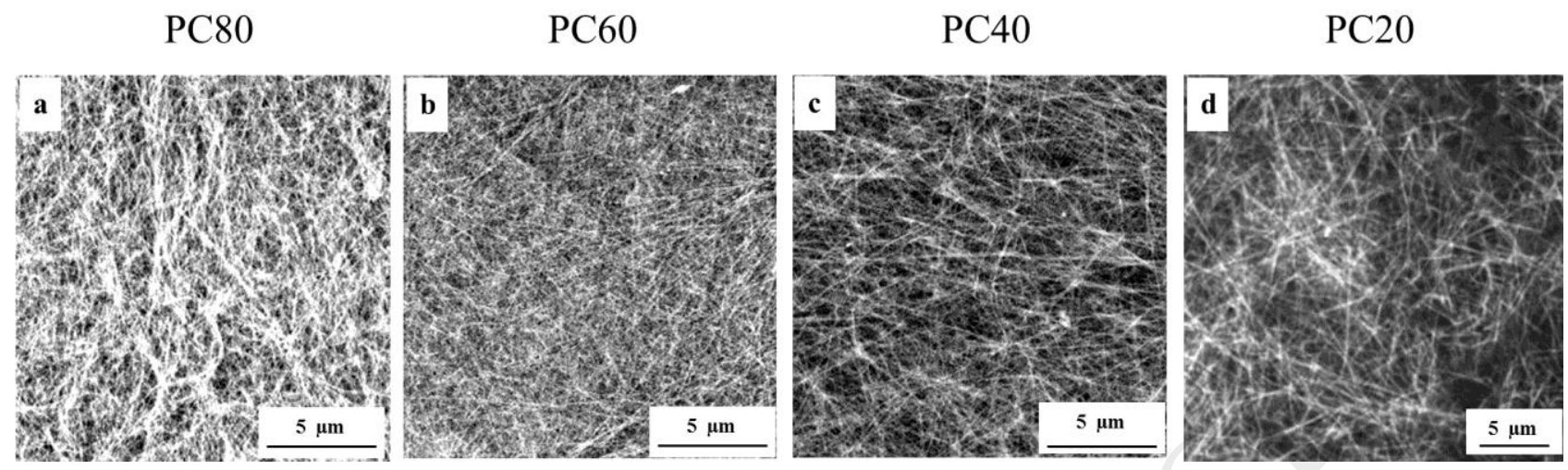

Fig. 2. Confocal reflectance microscopy of top collagen layer in compressed sheets of collagen-

PLGA-collagen with different collagen concentrations: a) PC80-PLGA-PC80, b) PC60-PLGAPC60, c) PC40-PLGA-PC40, d) PC20-PLGA-PC20.

TEM imaging was used to study the cross sectional appearance of different PC sheets and also to measure the thickness of each sheet after compression $\left(t_{p c}, F i g .1\right)$. According to TEM images (Fig. 3), a descending trend in the density of collagen fibrils along with decreasing collagen concentration in relevant hydrogels was observed. While PC80 exhibited very dense content of collagen fibrils (Fig. 3a), a dense but ordered arrangement of collagen fibrils was observed in PC60 (Fig. 3b). Moving towards PC20 (Fig. 3d), the density of fibrils was lesser to sparse.
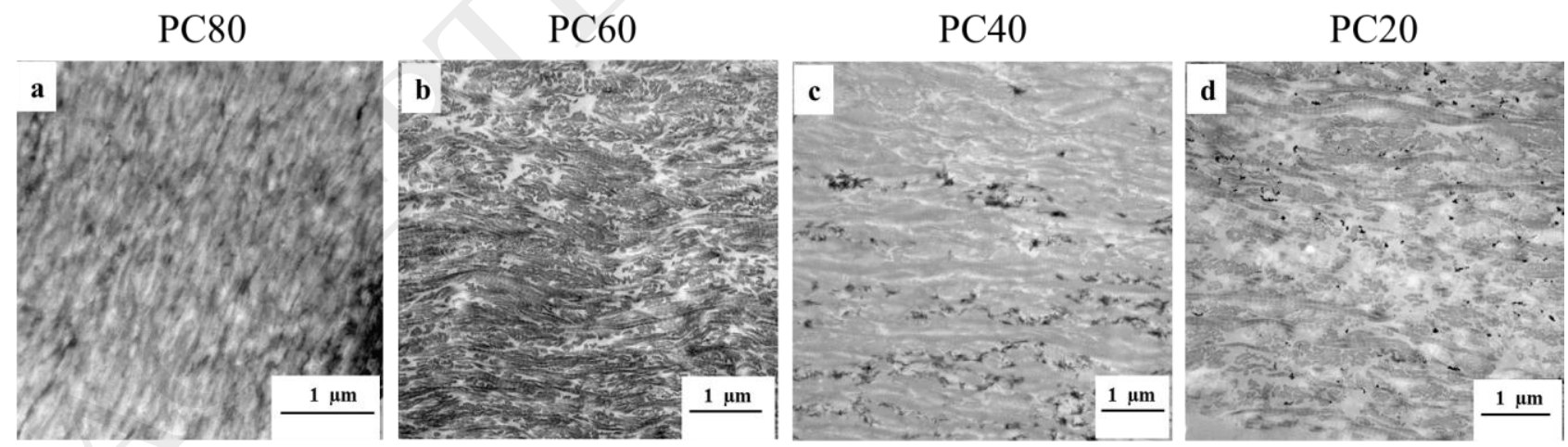

Fig. 3. Sectional imaging (TEM) of compressed collagen layer from different concentrations of collagen hydrogel, demonstrating decreasing density of collagen fibrils: a) PC80, b) PC60, c)

$$
\text { PC40, d) PC20 }
$$

Fig. 4a shows a cross-sectional image from a hybrid construct of PC80-PLGA-PC80 under light microscope, representing an integrated three-layered structure, in which the collagen layer had a 
thickness of around $5 \mu \mathrm{m}$ (Figs. $4 \mathrm{a}$ and b). By precise measurements of the compressed collagen layer in different constructs using TEM images, we observed that all hydrogels with the same initial dimensions retained similar dimensions after compression (Fig. 4c shows the cross section of PC20 in a PC20-PLGA-PC20 construct). Assuming that there would be no change in total collagen mass before and after compression, we calculated the collagen concentration in the final PC sheets (Equation 1). The results showed that PC20 had a fibrous network with collagen density of around $90 \mathrm{mg} / \mathrm{mL}$ (Table 2) compared to high-density collagen in PC80 (328 mg/mL, or 32\%). These results are of great importance, as we could generate PC sheets with distinctly lower concentration of collagen content, as represented by PC20, compared to conventional PC sheets, i.e. PC80, by just lowering the starting hydrogel concentration.

PC80-PLGA- PC80

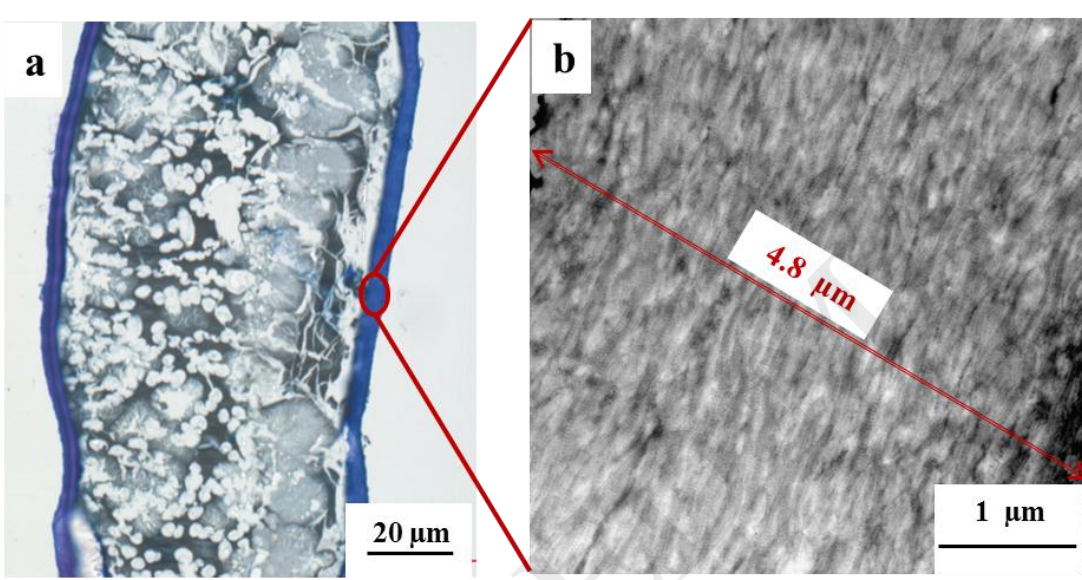

PC20

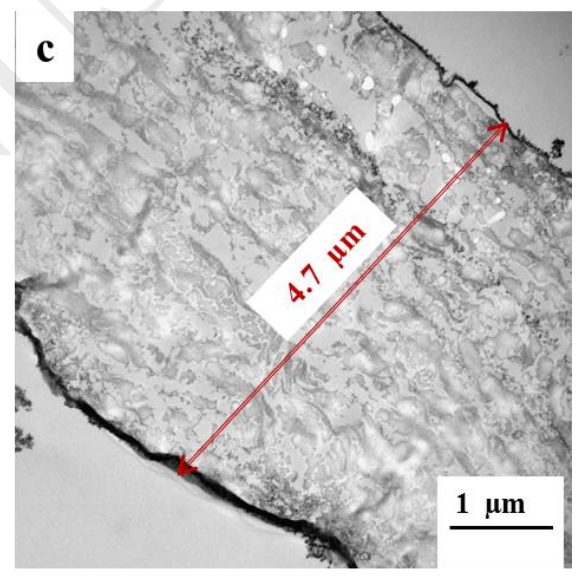

Fig. 4. Cross sectional view of the hybrid construct and the compressed sheets. a) Three layered structure of hybrid construct of collagen-PLGA-collagen showing two layers of collagen around electrosun sheet of PLGA, b) TEM section of PC80, and c) TEM section of PC20

Table 2: comparing collagen concentration in plastic compressed sheets with different initial collagen concentrations in hydrogel formula

\begin{tabular}{|c|c|c|c|c|c|}
\hline \multirow{4}{*}{ 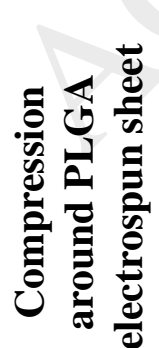 } & \multirow{2}{*}{$\begin{array}{l}\text { Plastic compressed } \\
\text { scaffolds }\end{array}$} & \multicolumn{2}{|c|}{ Collagen hydrogel thickness } & \multicolumn{2}{|c|}{$\begin{array}{c}\text { Collagen concentration } \\
(\mathrm{mg} / \mathrm{mL})\end{array}$} \\
\hline & & $\begin{array}{c}\text { Before } \\
\text { Compression } \\
(\mathrm{mm}) \\
\end{array}$ & $\begin{array}{c}\text { After } \\
\text { Compression } \\
(\mu \mathrm{m})\end{array}$ & $\begin{array}{c}\text { Before } \\
\text { compression }\end{array}$ & $\begin{array}{c}\text { After } \\
\text { compression }\end{array}$ \\
\hline & PC80-PLGA-PC80 & $1 \pm 0.1$ & $5 \pm 1.1$ & 1.64 & $328 \pm 23$ \\
\hline & PC60-PLGA-PC60 & $1 \pm 0.1$ & $4.7 \pm 0.95$ & 1.23 & $260 \pm 28$ \\
\hline & PC40-PLGA-PC40 & $1 \pm 0.1$ & $4.8 \pm 1.3$ & 0.82 & $188 \pm 25$ \\
\hline
\end{tabular}




$$
\begin{array}{l|llll}
\text { PC20-PLGA-PC20 } & 1 \pm 0.1 & 4.5 \pm 1.6 & 0.41 & 90 \pm 31
\end{array}
$$

Comparison with previous literature by Brown et al [6], demonstrated that compressing conventional collagen hydrogel (C80) with starting thickness of $3.6 \mathrm{~mm}$ and $91 \%$ of fluid loss leads to a sheet with average thickness of $21.8 \mu \mathrm{m}$ (collagen concentration of $270 \mathrm{mg} / \mathrm{mL}$ ), which is close to our PC60. Similarly, controlling the rate of water loss to around 87\% (decreasing compression time and applied load) resulted in a final thickness of $48.2 \mu \mathrm{m}(120 \mathrm{mg} / \mathrm{mL})$ in compressed sheet, which lies in a range between our PC20 and PC40. It is worth noting that compression procedure of conventional PC, can be engineered in terms of time and applied load so that final constructs of controllable densities are achieved [5,6]. However, due to immediate self-compression of collagen hydrogel, controlling such variables are difficult and would require time-consuming experiments in order to find an optimal formula. Therefore, we argue that altering the starting hydrogel collagen content, but not changing the other variables of the compression procedure as demonstrated in this study, is more realistic and reasonable.

\subsubsection{Tensile mechanical properties of hybrid scaffolds}

To compare tensile mechanical properties between PCX-PLGA-PCX (X =80,60, 40, 20), we performed tensile uniaxial rupture tests. Outcomes including elastic modulus, tensile strength (fracture stress), and elongation at break are listed in Table 3. Although PC20-PLGA-PC20 scaffolds represented highest modulus and tensile strength, as well as lowest elongation at break, the differences between the four scaffold types of PC80-PLGA-PC80, PC60-PLGA-PC60, PC40PLGA-PC40, and PC20-PLGA-PC20 were not significant. Considering that PLGA layer was similar in all four scaffold types and played the main role in maintaining the mechanical strength of the scaffold, we did not expect otherwise. We compared the mechanical properties of PC80-PLGAPC80 with PC80 in another study [18], and showed that PC80 is much weaker than hybrid construct of PC80-PLGA-PC80 in term of tensile strength (6 fold) and elongation at break (16 fold). It can be generalized to PC60, PC40, and PC20 as well to conclude why no significant difference between the different hybrid constructs (PCX-PLGA-PCX) was observed. 
Table 3: Tensile mechanical properties of hybrid constructs of PCX-PLGA-PCX (X = 80, 60, 40,

20)

\begin{tabular}{|c|ccc|}
\hline \multirow{2}{*}{ Hybrid constructs } & \multicolumn{3}{|c|}{ Tensile mechanical properties } \\
\cline { 2 - 4 } & Elastic modulus & Tensile strength & Elongation at break \\
& $(\mathrm{MPa})$ & $(\mathrm{MPa})$ & $(\%)$ \\
\hline PC80-PLGA-PC80 & $39.6 \pm 7.8$ & $3.84 \pm 1.26$ & $65 \pm 11$ \\
PC60-PLGA-PC60 & $35.5 \pm 12.7$ & $3.67 \pm 1.32$ & $57 \pm 8.4$ \\
PC40-PLGA-PC40 & $38.7 \pm 9.4$ & $3.95 \pm 0.96$ & $62 \pm 17.6$ \\
PC20-PLGA-PC20 & $46.1 \pm 11.5$ & $4.15 \pm 1.11$ & $51.5 \pm 13.8$ \\
\hline
\end{tabular}

\subsubsection{Nanomechanical characterization of compressed sheets}

We further characterized the nanomechanical properties of the compressed collagen sheets by means of AFM, in order to understand the structural and functional features of them as a variable of collagen concentration generated in this study. Force versus indentation curves were presented in Fig. 5(a). From these plots, various mechanical properties, including $E^{*}$ (Young's modulus, Figure $5(\mathrm{~b})), W_{\text {adh }}$ (work of adhesion, Figure 5(c)), and $E_{\text {Diss }}$ (dissipation energy, Figure 5(d)), were extracted as described in the section 2.2.3. The $E^{*}$ values of four different PC constructs were in the order of PC20 $(3.81 \pm 0.14 \mathrm{MPa})>\mathrm{PC} 40(0.89 \pm 0.09 \mathrm{MPa})>\mathrm{PC} 60(0.40 \pm 0.03 \mathrm{MPa})>\mathrm{PC} 80$ $(0.18 \pm 0.01 \mathrm{MPa})$. Overall, the observed $E^{*}$ values were in the same range with those of collagen hydrogels that were prepared by PC process and characterized by other methods than AFM [6,21]. It is most interesting to note that for the four samples, $E^{*}$ was inversely proportional to the final concentration of collagen hydrogels. This may appear surprising because for hydrogels, elasticity modulus is expected to be proportional to the amount of solid components. For example, a previous study by Hadjipanayi and colleagues [33] showed that Young's modulus of the collagen compressed sheets increased in proportion to the collagen content along a gradient after compression using a wedge-shaped mold. However, an important difference is that in that study, the collagen concentration ranged from 40 to $200 \mathrm{mg} / \mathrm{mL}$, whereas the present study involved 90 $\mathrm{mg} / \mathrm{mL}$ (C20, minimum) to $328 \mathrm{mg} / \mathrm{mL}$ (C80 maximum), i.e. the collagen concentration range is higher for the samples in this study. Our outcomes are however in agreement with Chieh and colleagues [23] where collagen hydrogel constructs with a lower initial collagen concentration (lower than $2 \mathrm{mg} / \mathrm{mL} ; 1.5,1$, and $0.5 \mathrm{mg} / \mathrm{mL}$ ) exhibited higher ultimate stress, and superior 
elasticity. We therefore assume that increased ratio of water to protein within the hydrogels which is maintained after compression also (please see the comparison in 3.1.1) may be considered as the factor improving the elasticity in the order of PC20 > PC40 > PC60 > PC80 (Fig. 5a). Moreover, we propose that the PLGA sheet in the middle of the collagen layers is a key factor that further assisted the enhancement of mechanical properties by controlled compressing of the collagen fibers. Accordingly, we observed (morphology shown by SEM and TEM images) more open collagen structure with C20 (lower initial density) and denser fibrous structure with PC80. In this sense, we can claim that an optimal collagen-based network was generated for PC20 with more distance between fibers and sustaining elastic mechanical structure. Weaker mechanical properties with increasing density of collagen were shown by greater indentation to achieve the similar $F / R$ normal force values (Fig. 5(a)). The decreasing elasticity with increasing collagen density was accompanied with increasing $W_{\text {adh }}$ and $E_{\text {diss, }}$, except a reversed trend between C60 and C80 in $W_{\text {adh }}$. This might be related to that the opposing surface is $\mathrm{PE}$, a hydrophobic probe, that hydrophobic interaction is responsible for the adhesive forces between AFM probe and collagen samples.


Fig. 5. (a) F/R vs. Indentation depth ( $\mu \mathrm{m})$ for the samples by means of AFM (PE probe). (b) $E^{*}$ (c) $\mathrm{W}_{\text {adh }}(\mathrm{d}) \mathrm{E}_{\mathrm{diss}}$ for PC20, PC40, PC60 and PC80 


\subsection{Cell-scaffold interactions}

\subsubsection{Cell attachment}

Lastly, we compared cell-scaffold behaviors between hybrid constructs of PCX-PLGA-PCX by seeding fibroblasts onto the scaffolds and studied cell attachment, metabolic activity, and proliferation by SEM, MTS and cell nuclei counting. SEM images of cell-seeded scaffolds after 7 days in culture (Fig. 6) showed that all types of scaffolds supported cell attachment. Cells were spread uniformly on all scaffold types, but the morphological appearance presented significant differences. For instance, for PC80 (Fig. 6a), a dense 2D-like structure was observed, whereas for PC60 (Fig. 6b), more 3D structure and different layers of fibers were visible (the second row in Fig. 6 shows images with higher resolution). PC40 (Fig. 6c), not only confirmed a 3D structure, but also that some cells (shown in red circle) were covered by fibers. In PC20 surface (Fig. 6d), an open 3D structure was shown with collagen fibers crossing over the cells (red circles in Fig. 6d second raw).
(a) PC80-PLGA-PC80
(b) PC60-PLGA-PC60
(c) PC40-PLGA-PC40
(d) PC20-PLGA-PC20
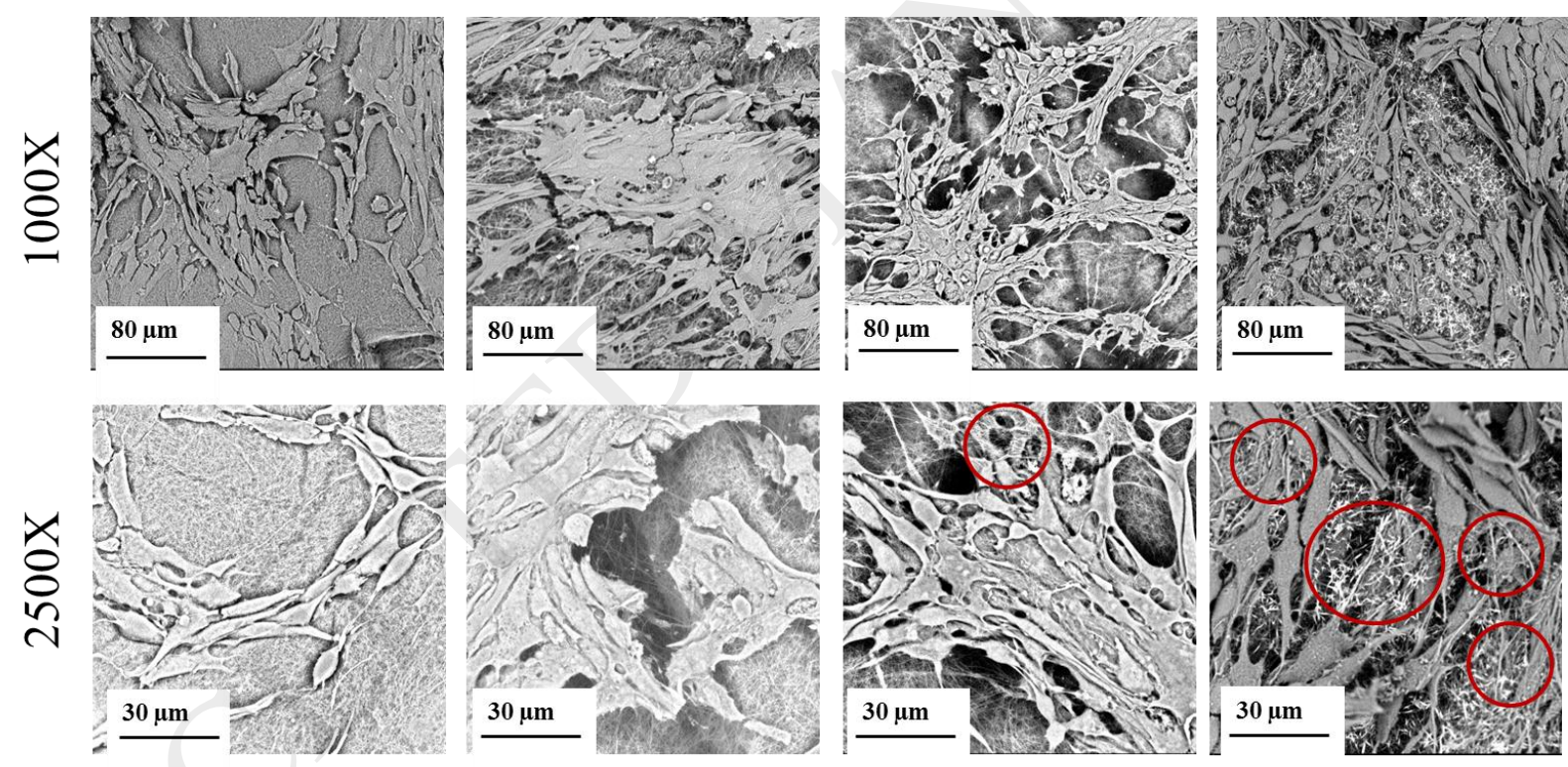

Fig. 6. SEM images of $3 \mathrm{~T} 3$ fibroblasts after 7 days culturing onto PCX-PLGA-PCX constructs under two different (1000x, 2500X) magnifications: a) PC80-PLGA-PC80, b) PC60-PLGA-PC60,

c) PC40-PLGA-PC40, d) PC20-PLGA-PC20

\subsubsection{Metabolic activity, proliferation and infiltration of cells}

The metabolic activity of cells that were included in the hybrid constructs were determined by a MTS assay after cell expansion on the scaffolds over a period of 7 days (Fig. 7a). It was found that 
the metabolic activity of cells on all hybrid constructs increases with culture time. We also observed that although there were no significant differences between four constructs after 1 day in culture, PC80 and PC20 had the lowest and highest metabolic activities respectively after 4 and 7 days in culture. In general, there was a progressive trend in metabolic activity of cells from PC80 to PC20, but the difference was insignificant in some cases.

Cell proliferation studies were performed by counting the number of adhered cells (cell nuclei stained by DAPI) on different constructs of PCX-PLGA-PCX (Fig. 7b). It was again shown that there was no significant difference between the number of adherent cells after 1 day in culture. But, an increasing trend in number of adherent cells (from PC80 to PC20) after 7 days in culture was observed.

a

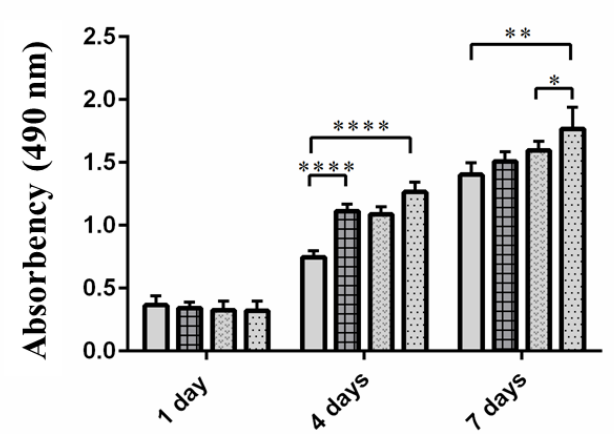

Days in culture b

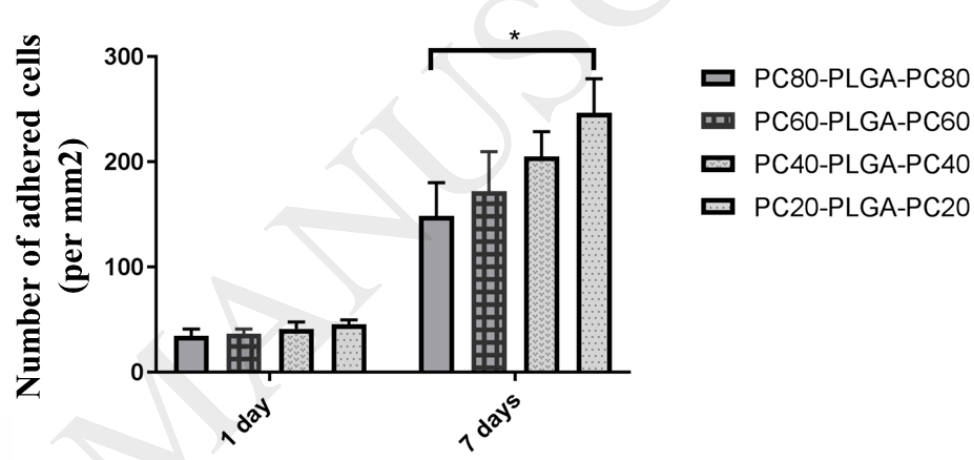

Days in culture

Fig. 7. Viability and proliferation of $3 \mathrm{~T} 3$ fibroblasts on different layered constructs: (a) metabolic activity of 3T3 fibroblasts on PC80-PLGA- PC80, PC60-PLGA-PC60, PC40-PLGA-PC40, and PC20-PLGA-PC 20 measured by an MTS assay, (b) proliferation of 3T3 fibroblasts on PC80PLGA- PC80, PC60-PLGA-PC60, PC40-PLGA-PC40, PC20-PLGA-PC20 measured by counting number of cell nuclei stained by DAPI.

The confocal microscope images (Fig. 8) revealed an increased spatial density of cells from PC80 to PC20. Moreover, orthogonal images from PC20 and PC40 (Figs. 8 c, d) showed infiltration of cells into the collagen layer making multi-cellular layers (cell nuclei stained blue with DAPI) compared to single layer of fibroblasts in PC80-PLGA-PC80. 
PC80-PLGA- PC80

PC60-PLGA-PC60

PC40-PLGA-PC40

PC20-PLGA-PC 20
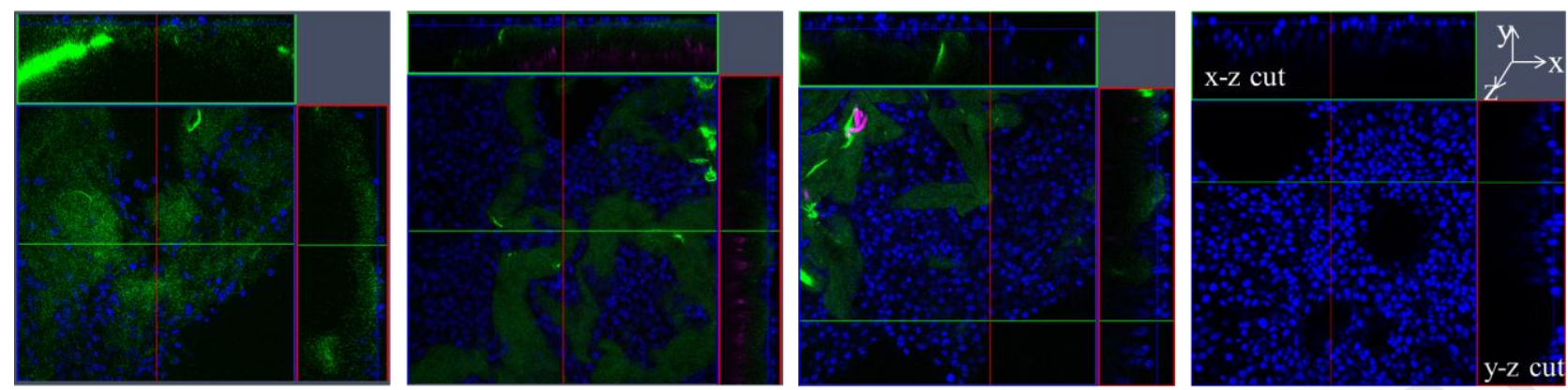

Fig. 8. Fluorescent confocal micrographs of PC80-PLGA-PC80, PC60-PLGA-PC60, PC40-PLGA-

PC40, and PC20-PLGA-PC20 scaffolds seeded by 3T3 fibroblasts and cultured in vitro for 7 days, including orthogonal edge views. It demonstrates sparse single layer of cells on PC80-PLGA-PC80 scaffold, into a multilayered dense cellular construct in PC20-PLGA-PC20. Samples are fixed and stained with DAPI (blue) on cells, AlexaFluor 488 phalloidin (green) on collagen, and Rhodamine

$$
\text { B (red) on PLGA. }
$$

Overall, we have shown that by decreasing the initial collagen concentration in the collagen hydrogel formula from around $1.64 \mathrm{mg} / \mathrm{mL}$ to around $0.41 \mathrm{mg} / \mathrm{mL}$, not only the consumption of collagen was decreased, but also a collagen structure that was less dense than conventional PC sheets (PC80), yet providing more favorable cell activities, was achieved.

To our knowledge there are no similar studies aiming at reducing the density of compressed collagen sheets by altering the initial collagen concentration. However, some studies have aimed at improving the mechanical properties through increasing the collagen concentration in sheets of PC. For instance, Bitar and colleagues[4], studied the effect of multiple compression on cellular dense collagen scaffolds through serial compression and showed a dense and predominantly viable cell population in the single compressed (SC) collagen sheet at day 10 in culture, whilst clear signs of cell death were observed in double compressed sheets (DC) at the same time point. These studies further demonstrate the negative impact that an increased collagen density has on cell viability. In another study, Hadjipanayi and colleagues [21] compressed a wedge-shaped collagen construct to produce a sheet of uniform thickness, but with increasing collagen density along the length of the sheet. They reported preferential migration of human dermal fibroblasts towards the denser part of the gradient after 3 and 6 days in culture, concluding that cells proliferated faster when seeded within compressed sheets with higher density. In contrast, the present study revealed that lower density collagen sheets represent higher cell viability and proliferation. Their outcomes could be in 
agreement with ours if we consider it from another point of view, which is their migration tendency. In other words, the lower density collagen sheets in the study of Hadjipanayi and colleagues [21], provided cells with more freedom to infiltrate in depth of scaffold toward the dense region. They were then trapped in high-density PC sheets. Helary and colleagues[11] also studied the effect of collagen hydrogel concentration on cell morphology, remodeling, and proliferation of human myofibroblasts. They showed that dense collagen matrices $(40 \mathrm{mg} / \mathrm{ml})$ supported better proliferation and differentiation of myofibroblasts. Cells within dense collagen matrices $(40 \mathrm{mg} / \mathrm{ml})$ were described as spindle shaped, and similar to cells observed during tissue repair, whilst cells within loose matrices $(5 \mathrm{mg} / \mathrm{ml})$ were more rounded. It may appear contradictory to our conclusion. However, the point is that Helary and colleagues have applied collagen hydrogels (not compressed sheets). If we consider the comparison based on collagen concentration, we see that our PC20 (collagen concentration of $90 \mathrm{mg} / \mathrm{mL}$ ) has the closest concentration of collagen (among PC sheets) to the optimum collagen hydrogel $(40 \mathrm{mg} / \mathrm{mL})$ in the study performed by Helary et al. A comparison between conventional collagen hydrogels, and compressed hydrogels for corneal tissue engineering has also been performed by $\mathrm{Mi}$ and colleagues[12]. They demonstrated the superiority of compressed collagen gel to conventional collagen gels for the construction of an artificial corneal epithelium, due to structural similarity of compressed gels to the normal corneal stroma. In total, it seems that PC20 can be considered as an optimal collagen sheet for different tissue engineering applications, especially those requiring higher rates of cell migration and elasticity. We can however select any of the other constructs (PC40, PC60, and PC80) or something in between them based on the cellular or biomechanical requirements of the final target tissue. Further advantages of PC sheets in comparison to hydrogels include higher mechanical strength, customizable dimensions, ease of manipulation, and possibility of fixation with forceps and sutures.

\section{Conclusions}

We have shown in this study that we can easily control the final protein content and fibrillar density of compressed collagen sheets by altering the starting hydrogel collagen formula compared to conventional hydrogels applied for PC of collagen. It was observed that increasing the collagen density in PC sheets suppresses the proliferation and metabolic activity of fibroblasts, as well as elasticity of the scaffolds. The technique is simple, fast, and realistic. Moreover, there is no need to change other variables of the compression procedure. 


\section{Author Contributions}

Conceived and designed the experiments: FA MF SL ISC. Performed the experiments: FA AA NN. Analyzed the data: FA AA SL. Wrote the paper: FA. All authors reviewed and edited the article before submission

\section{Acknowledgments}

This work was supported by grants from Danish Research Council Foundation (Individual Postdoctoral grant 4093-00282A, and Sapere Aude: DFF-Research Talent 4217-00048A).

\section{References}

[1] A. Asti, L. Gioglio, Natural and synthetic biodegradable polymers: different scaffolds for cell expansion and tissue formation., Int. J. Artif. Organs. 37 (2014) 187-205. doi:10.530/ijao.5000307.

[2] J. Glowacki, S. Mizuno, Collagen Scaffolds for Tissue Engineering Collagen Scaffolds for Tissue Engineering, Biopolymers. 89 (2007) 338-344. doi:10.1002/bip.20871.

[3] E.A. Abou Neel, U. Cheema, J.C. Knowles, R.A. Brown, S.N. Nazhat, Use of multiple unconfined compression for control of collagen gel scaffold density and mechanical properties, Soft Matter. 2 (2006) 986-992. doi:10.1039/b609784g.

[4] M. Bitar, V. Salih, R. a. Brown, S.N. Nazhat, Effect of multiple unconfined compression on cellular dense collagen scaffolds for bone tissue engineering, J. Mater. Sci. Mater. Med. 18 (2007) 237-244. doi:10.1007/s10856-006-0685-1.

[5] U. Cheema, R.A. Brown, Rapid Fabrication of Living Tissue Models by Collagen Plastic Compression : Understanding Three-Dimensional Cell Matrix Repair In Vitro, Adv. WOUND CARE. 2 (2013) 176-184. doi:10.1089/wound.2012.0392.

[6] R. a. Brown, M. Wiseman, C.-B. Chuo, U. Cheema, S.N. Nazhat, Ultrarapid Engineering of Biomimetic Materials and Tissues: Fabrication of Nano- and Microstructures by Plastic Compression, Adv. Funct. Mater. 15 (2005) 1762-1770. doi:10.1002/adfm.200500042.

[7] J. Tien, C.M. Nelson, C.S. Chen, Fabrication of aligned microstructures with a single elastomeric stamp., Proc. Natl. Acad. Sci. U. S. A. 99 (2002) 1758-1762. doi:10.1073/pnas.042493399.

[8] K.M. Chrobak, D.R. Potter, J. Tien, Formation of perfused, functional microvascular tubes in vitro, Microvasc. Res. 71 (2006) 185-196. doi:10.1016/j.mvr.2006.02.005.

[9] C.R. Lee, a. J. Grodzinsky, M. Spector, The effects of cross-linking of collagen-glycosaminoglycan scaffolds on compressive stiffness, chondrocyte-mediated contraction, proliferation and biosynthesis, Biomaterials. 22 (2001) 3145-3154. doi:10.1016/S0142-9612(01)00067-9.

[10] S. Ramanujan, A. Pluen, T.D. McKee, E.B. Brown, Y. Boucher, R.K. Jain, Diffusion and convection in collagen gels: implications for transport in the tumor interstitium., Biophys. J. 83 (2002) 1650-1660. doi:10.1016/S0006-3495(02)73933-7.

[11] C. Helary, L. Ovtracht, B. Coulomb, G. Godeau, M.M. Giraud-Guille, Dense fibrillar collagen matrices: A model to study myofibroblast behaviour during wound healing, Biomaterials. 27 (2006) 4443-4452. doi:10.1016/j.biomaterials.2006.04.005.

[12] S. Mi, B. Chen, B. Wright, C.J. Connon, Plastic compression of a collagen gel forms a much improved scaffold for ocular surface tissue engineering over conventional collagen gels, J. Biomed. Mater. Res. - Part A. 95A (2010) 447-453. doi:10.1002/jbm.a.32861. 
[13] H.J. Levis, R.A. Brown, J.T. Daniels, Biomaterials Plastic compressed collagen as a biomimetic substrate for human limbal epithelial cell culture, Biomaterials. 31 (2010) 7726-7737. doi:10.1016/j.biomaterials.2010.07.012.

[14] H.J. Levis, G.S.L. Peh, K. Toh, R. Poh, A.J. Shortt, R.A.L. Drake, J.S. Mehta, J.T. Daniels, Plastic Compressed Collagen as a Novel Carrier for Expanded Human Corneal Endothelial Cells for Transplantation, PLoS One. 7 (2012). doi:10.1371/journal.pone.0050993.

[15] S. Ashraff, R.A. Brown, J.B. Phillips, Plastic compression of collagen : development and assessment of a new biomaterial in nerve repair, Eur. Cells Mater. 10 (2005) 35.

[16] E.-M. Engelhardt, L. a Micol, S. Houis, F.M. Wurm, J. Hilborn, J. a Hubbell, P. Frey, A collagenpoly(lactic acid-co- $\varepsilon$-caprolactone) hybrid scaffold for bladder tissue regeneration., Biomaterials. 32 (2011) 3969-76. doi:10.1016/j.biomaterials.2011.02.012.

[17] F. Ajalloueian, S. Zeiai, R. Rojas, M. Fossum, J. Hilborn, One-Stage Tissue Engineering of Bladder Wall Patches for an Easy-To-Use Approach at the Surgical Table, Tissue Eng. Part C. Methods. 19 (2013) 688-696. doi:10.1089/ten.tec.2012.0633.

[18] F. Ajalloueian, S. Zeiai, M. Fossum, J.G. Hilborn, Constructs of electrospun PLGA, compressed collagen and minced urothelium for minimally manipulated autologous bladder tissue expansion., Biomaterials. 35 (2014) 5741-8. http://www.ncbi.nlm.nih.gov/pubmed/24768046.

[19] P. Sawadkar, S. Alexander, V. Mudera, Tissue-engineered collagen grafts to treat large tendon defects., Regen. Med. 9 (2014) 249-51. doi:10.2217/rme.14.15.

[20] E. Engelhardt, E. Stegberg, R.A. Brown, J.A. Hubbell, F.M. Wurm, M. Adam, P. Frey, Compressed collagen gel : a novel scaffold for human bladder cells, J. Tissue Eng. Regen. Med. 4 (2010) 123130. doi:10.1002/term.

[21] K. Hu, H. Shi, J. Zhu, D. Deng, G. Zhou, W. Zhang, Y. Cao, W. Liu, Compressed collagen gel as the scaffold for skin engineering, Biomed Microdevices. 12 (2010) 627-635. doi:10.1007/s10544-0109415-4.

[22] E.L. Abreu, M.P. Palmer, M.M. Murray, Collagen density significantly affects the functional properties of an engineered provisional scaffold, J Biomed Mater Res A. 93 (2010) 150-157. doi:10.1002/jbm.a.32508.

[23] H.-F. Chieh, Y. Sun, J.-D. Liao, F.-C. Su, C. Zhao, P.C. Amadio, K.-N. An, Effects of cell concentration and collagen concentration on contraction kinetics and mechanical properties in a bone marrow stromal cell-collagen construct, J Biomed Mater Res A. 93 (2010) 1132-1139. doi:10.1002/jbm.a.32606.

[24] J.E. Sader, J. Pacifico, C.P. Green, P. Mulvaney, General scaling law for stiffness measurement of small bodies with applications to the atomic force microscope, J. Appl. Phys. 97 (2005) 124903. doi:10.1063/1.1935133.

[25] J.E. Sader, J.A. Sanelli, B.D. Adamson, J.P. Monty, X. Wei, S.A. Crawford, J.R. Friend, I. Marusic, P. Mulvaney, E.J. Bieske, Spring constant calibration of atomic force microscope cantilevers of arbitrary shape, Rev. Sci. Instrum. 83 (2012) 103705. doi:10.1063/1.4757398doi.org/10.1063/1.4757398.

[26] K.L. Johnson, K. Kendall, A.D. Roberts, K.L. Johnsont, Surface Energy and the Contact of Elastic Solids, Proc. R. Soc. London. 324 (1971) 301-313. http://www.jstor.org/stable/78058 (accessed February 9, 2017).

[27] L. Sirghi, F. Rossi, The effect of adhesion on the contact radius in atomic force microscopy indentation, Nanotechnology. 20 (2009) 1-8. doi:10.1088/0957-4484/20/36/365702.

[28] Y. Sun, B. Akhremitchev, G.C. Walker, Using the Adhesive Interaction between Atomic Force Microscopy Tips and Polymer Surfaces to Measure the Elastic Modulus of Compliant Samples, Langmuir. 20 (2004) 5837-5845. doi:10.1021/la036461q.

[29] A.C. Mendes, N. Nikogeorgos, S. Lee, I.S. Chronakis, Nanomechanics of electrospun phospholipid fiber, Appl. Phys. Lett. 106 (2015) 223108. doi:10.1063/1.4922283.

[30] J. Grosst, D. Kirks, The Heat Precipitation of Collagen from Neutral Salt Solutions: Some RateRegulating Factors \&quot;, J. Biol. Chem. 233 (1958). 
[31] V.L. Cross, Y. Zheng, N.W. Choi, S.S. Verbridge, B.A. Sutermaster, L.J. Bonassar, C. Fischbach, A.D. Stroock, Dense type I collagen matrices that support cellular remodeling and microfabrication for studies of tumor angiogenesis and vasculogenesis in vitro, Biomaterials. 31 (2010) 8596-8607. doi:10.1016/j.biomaterials.2010.07.072.

[32] R.M. Kuntz, W.M. Saltzman, Neutrophil Motility in Extracellular Matrix Gels: Mesh Size and Adhesion Affect Speed of Migration, Biophys. J. 72 (1997) 1472-1480.

[33] E. Hadjipanayi, V. Mudera, R. a. Brown, Guiding cell migration in 3D: A collagen matrix with graded directional stiffness, Cell Motil. Cytoskeleton. 66 (2009) 121-128. doi:10.1002/cm.20331. 